\title{
Disposition ascriptions
}

\author{
Simona Aimar ${ }^{1}$
}

Published online: 26 March 2018

(C) The Author(s) 2018

\begin{abstract}
I argue that disposition ascriptions—claims like 'the glass is fragile' — are semantically equivalent to possibility claims: they are true when the given object manifests the disposition in at least one of the relevant possible worlds.
\end{abstract}

Keywords Dispositional adjectives · Disposition ascriptions · Modality · Possibility claims · Dispositions · Gradable adjectives · Manifestations

\section{Introduction}

Some things are fragile. Some people are irascible. We often ascribe dispositions to objects. But when is a given disposition ascription true? For instance, when are claims such as

(1) The glass is fragile.

(2) Bill is irascible.

true?

In this paper I put forward the view that disposition ascriptions (henceforth, DAs) are semantically equivalent to possibility claims, and thus can be understood as introducing existential quantification over a given set of possible worlds. For instance, (1) is true just in case there is at least one relevant possible world in which the glass gets broken easily. Call this the Possibility View.

The standard counterfactual account of DAs is significantly different from the Possibility View. It interprets (1)-(2) as concealed counterfactual conditionals (see, inter alia, Goodman 1954; Quine 1960). For instance, it says that (1) is true just in

Simona Aimar

s.aimar@ucl.ac.uk

1 University College London, London, UK 
case, if the glass were struck, it would break. Notoriously, the account faces several counterexamples. So-called finks, masks and reverse-finks, among others, put the counterfactual account in a corner. ${ }^{1}$

Faced with counterexamples, one has at least two options. One option is to try and develop a more sophisticated version of the standard account. ${ }^{2}$ But whether some sort of counterfactual account of DAs will ultimately be successful remains to be seen. A second option is to conclude that the prospect for counterfactual accounts of DAs look grim. From this perspective, counterexamples provide us with motivation for a change of route. This paper puts forward one such alternative route-the Possibility View. ${ }^{3}$

Some authors have already started to make steps towards options in the vicinity of the Possibility View. Manley and Wassermann (2007, 2008) suggest that something has a given disposition just in case it manifests that disposition in a suitable proportion of relevant cases. They thereby connect the possession of dispositions with a modal force weaker than necessity. Vetter $(2014,2015)$ claims that several DAs have a modal force close to standard possibility. For instance, to her (1) is true just in case the glass breaks in a relatively low proportion of cases. She adds that some DAs, like 'the glass is breakable', are possibility claims. Yet neither of these authors goes as far as to see that all DAs are actually possibility claims-as the Possibility View says. Within the linguistics literature, Angelika Kratzer $(1981,2012,2013)$ suggests in passing that something along the lines of the Possibility View might be correct. This paper develops such a view, offers independent and novel arguments in its support, and shows why it scores better than alternatives in its vicinity.

I first elucidate the basic tenets of the Possibility View and give an argument to motivate its pursuit (Sects. 2-4). Then I develop the view in detail (Sects. 5-8). I conclude by addressing two potential objections (Sect. 9).

\footnotetext{
${ }^{1}$ A simple counterexample to the standard view which counts as a mask case: a glass is packed in such a way that if it were struck it would not break; even so, the glass seems fragile. Since the standard counterfactual account says that the glass is fragile just in case it would break when struck, the glass comes out as not fragile. For further counterexamples see Martin (1994), Bird (1998), and Contessa (2016), inter alia.

${ }^{2}$ Cf. Lewis (1997a), Choi (2006), Bonevac et al. (2011) and Contessa (2013, 2016). For objections, see inter alia Bird (1998), Yli-Vakkuri (2010), Manley and Wassermann (2008) and Vetter (2011, 2014, 2015).

${ }^{3}$ For an alternative that is distant from the Possibility View, see Fara (2005), where DAs are regarded as equivalent to habituals; for an objection, see Yli-Vakkuri (2010).

${ }^{4}$ Shortly before publishing this paper, I was informed that Maier (forthcoming) suggests an account of DAs which is also in the vicinity of the Possibility View; I am sorry I cannot compare and contrast our two views in the present piece. We both presented independently achieved results on DAs at the Workshop on the Morphological, Syntactic and Semantic Aspects of Dispositions in Stuttgart in June 2015. For other interesting papers given at that conference, see Martin et al. (2016).
} 


\section{The view and its scope}

The expression 'disposition ascription' is not used homogeneously in the debate. Here is a use of 'disposition ascription' I do not adopt in this paper. Some authors call claims of the form ' $x$ is disposed to $\varphi$ ' disposition ascriptions. ${ }^{5}$ The reason I will not follow this trend is the following. In the literature, the expression '...disposed to...' is a term of art. It is meant to capture whatever feature an object has in virtue of its possessing a given disposition. ${ }^{6}$ My goal here is to shed light on ordinary dispositional talk. So I will not consider claims of the form ' $x$ is disposed to $\varphi$ '. This way I will be sure not to rely on theoretically based intuitions that may come with these terms of art.

Other authors point out that ordinary dispositional talk involves adjectives such as 'fragile', 'irascible' and 'breakable'. ' I agree with this suggestion, and assume as a rule of thumb that any adjective ending in -ile/-able/-ible is a dispositional adjective. $^{8}$ So on my terminology claims such as sentences of the form

(DA) $x$ is F-able/-ible/-ile.

are disposition ascriptions (DAs).

The account of DAs I put forward is simple enough. It says that all DAs express possibility, because dispositional adjectives are possibility modals. Given a possible world semantics:

(Possibility View) ' $x$ is F-able' is true iff there is at least one accessible possible world in which $x$ (-es. ${ }^{9}$

Here ' $x$ ' picks up the subject of the DA, 'F-able' is the relevant dispositional adjective, and ' $\varphi$ ' describes the manifestation of the disposition corresponding to the dispositional adjective (without necessarily being the lexically correlated infinitive). The qualification 'accessible' indicates that the context selects the modal

\footnotetext{
5 The trend starts with Lewis (1997a), who is explicitly not interested with ordinary language DAs. Amongst followers, see Bird (1998), Fara (2005), Manley and Wassermann $(2007,2008)$ and Steinberg (2010). Before Lewis, DAs were at the centre of the scene-cf. Goodman (1954), Quine (1960, 1970), and Mackie (1972), inter alia.

6 As Vetter (2015: 67) points out, 'disposed to' often means something like 'willingly', or expresses statistical probability.

7 See esp. Vetter (2014, 2015: 63ff).

8 I consider cases like 'possible' and 'probable' to be exceptions to this rule, and follow the literature in assuming that they deserve their own special treatment. I also ignore adjectives whose meaning has been theoretically stipulated, such as 'countable' in mathematics (a set, say, is countable just in case it has the same cardinality of some subset of the set of natural numbers).

I leave the task of investigating whether further adjectives should qualify as dispositional to future work. It should also be mentioned that in linguistics the expression 'dispositional sentences' is used differently than in this paper: it is taken to refer to generic sentences such as 'the printer prints 100 pages per minute'—cf. Menéndez-Benito (2013). Interestingly enough, the view I am about to put forward for DAs here is analogous to the view Menéndez-Benito puts forward for the relevant class of generic sentences.

9 Kratzer (2007) makes a case for using possible situations instead of possible worlds. In the main text, I use possible worlds for simplicity—so does Kratzer (1981, 1991, 2012, 2013), inter alia.
} 
base, namely which possible worlds are accessible from the world of evaluation. In other words: $x$ is F-able just in case there is an accessible possible world in which $x$ manifests the given disposition. For instance, consider the claim:

(3) The glass is breakable.

(3) is true just in case there is at least one accessible possible world at which the glass manifests breakability—namely, it gets broken. Which worlds are accessible depends on context (see Sect. 5).

Note that the view assumes that there is always an associated verb phrase for each dispositional predicate. It does not assume that the verbal phrase has to be etymologically on a par with the predicate in question. It also does not assume that it is always obvious what that phrase is - at times, some work is needed to figure this out (see Sects. 6-9). ${ }^{10}$

It is worth emphasising from the outset that the Possibility View is not a metaphysical thesis about dispositions. It does not imply, for instance, that dispositions are reducible to possibility. Nor is it a metaphysical thesis about modality. It does not imply, for instance, that modality is to be grounded in dispositions. ${ }^{11}$ Moreover, the view by itself does not settle whether we should include dispositions and modality in our metaphysics. Rather, the Possibility View is a semantic thesis. It clarifies how to understand the DAs that one finds in ordinary language.

At the same time, the Possibility View does clarify some of the central assumptions that ordinary speakers make about dispositions when assessing DAs. For instance, it clarifies that speakers assume-either at an explicit or at an implicit level - that dispositions have manifestations. Accordingly, the Possibility View can provide us with a starting point to think about dispositions. Thus if one assumes that, other things being equal, a metaphysical account of dispositions should accommodate the assumptions that ordinary speakers make about dispositions, then the Possibility View is also a first step towards an account of dispositions. I leave the potential task of developing such an account to future occasions. ${ }^{12}$

\subsection{Immediate implications}

Before expanding and defending the Possibility View, it is worth pausing for a moment to spell out its immediate implications.

\footnotetext{
${ }^{10}$ We will see how the correct characterization of the manifestation of fragility, for instance, requires a verbal phrase that also contains adverbial qualifications (see Sects. 2.1, 6). Moreover, for some dispositional adjectives it is opaque what the associated verbal phrase is (e.g. durable, sizeable) but this can nonetheless be recovered. To pick the case of 'durable', for instance: roughly, something is durable just in case it lasts long enough (note that the Latin word for 'to last' is 'durare'). Finally, at times the modal force of some of these dispositional adjectives may appear stronger than possibility (consider for instance 'the bill is payable'). I discuss this last kind of cases in Sect. 9.1.

11 Borghini and Williams (2008) and Vetter (2015) argue that the metaphysics of modality is grounded in dispositions. Borghini and Williams (2008) do not discuss the semantics of DAs which is the focus of this paper. Vetter $(2014,2015)$ does consider the semantics of DAs-I discuss her view in Sects. 7.2-8.1.

12 I provide an account of dispositions that is in line with the Possibility View in Aimar (ms).
} 
The first immediate implication of the Possibility View is the following. If $x$ is F-able, there is at least one world in which $x \varphi$-es. In other words: if $x$ has a disposition, there is at least one relevant possible world in which $x$ manifests that disposition. Note that the view does not say that something that has a disposition at a world also manifests that disposition at that very world. So it allows that claims such as

(4) The glass is fragile even though it actually never breaks.

can be true-as seems plausible. What the view does rule out is that dispositions may necessarily lack manifestations. It assumes that, in the relevant circumstances, dispositions can be manifested.

The second immediate implication of the Possibility View is the following. If $x$ manifests a given disposition in an accessible possible world, then $x$ has that disposition in the evaluation world. This follows from the reflexivity of the accessibility relation. The implication further entails:

(The sufficiency of manifestations thesis): If $x$ actually manifests a disposition, $x$ actually has that disposition.

For instance, consider breakability. The sufficiency of manifestations thesis tells us that if a glass actually breaks, then it is breakable.

Note that the sufficiency of manifestations thesis sets the Possibility View apart from virtually all contemporary accounts of dispositional expressions. ${ }^{13}$ There is a reason authors typically deny this sufficiency thesis: it clashes with their coarse-grained picture of manifestations. Typically, breaking is regarded as the manifestation of fragility. Yet not everything that breaks is fragile. Thus scholars submit that manifesting fragility is not enough for something to count as fragile_pace the sufficiency of manifestations thesis. So why does the Possibility View endorse this thesis?

This is important. Instead of discharging the sufficiency of manifestations thesis, the defender of the Possibility View chooses to discharge something else instead. On the faces of cases like fragility, she concludes that we need a more fine-grained account of manifestations. For example, since not everything that breaks is fragile, for the defender of the Possibility View the manifestation of fragility is not breaking, but a more fine-grained event, such as the event of breaking easily. ${ }^{14}$ Accordingly, she can accept that, if something manifests fragility (i.e. it breaks easily), it is fragile. As a result, the Possibility View both preserves the sufficiency of manifestations thesis and accounts for the semantic difference one finds between adjectives such as 'fragile' and 'breakable'. These two adjectives differ in meaning because they are associated with different manifestation events (for details, see Sect. 6).

Finally, the Possibility View does not say that a disposition-bearer needs to have a given disposition at the same time of its manifestation. Usually this is the case. Yet

\footnotetext{
13 For some of the most explicit denials of the sufficiency of manifestations thesis, in connection with dispositions such as fragility and irascibility, see Vetter (2015: 70ff), Manley and Wasserman (2008: 74ff), and Fara (2005). Counterfactual accounts of DAs deny the thesis in virtue of their equating DAs with restricted necessity claims.

14 This paper assumes that breaking easily is a more specific event than breaking, in line with a neoDavidsonian event semantics.
} 
in some cases it isn't. Consider a flammable entity. Plausibly, the entity loses its flammability while burning and turning into ashes. Its manifesting the disposition shows that it had the disposition when the burning started to occur, but not that it preserved the disposition while manifesting it. Similarly, some dead stars are still visible to us: they manifest their visibility also after they cease to exist. So a disposition-bearer does not have to always exist at the time in which the disposition gets manifested. The Possibility View is compatible with scenarios of this kind.

\section{The bare bones of the argument}

The view that DAs express possibility has some intuitive plausibility, but remains far from trivial. I put forward an extensive and novel argument for the view. My argument consists of three main parts.

First, I offer reasons for considering the Possibility View a plausible one (Sect. 4). I give a general argument for why one should think that DAs express possibility. The argument takes the form of an inference to the best explanation. Here is its general form: within embedded contexts, the affirmation (or denial) of a DA appears to entail a given possibility claim, and vice versa. The simplest explanation of this phenomenon is that DAs express possibility. So we have reason to conclude that DAs express possibility.

Having established that the Possibility View is worth pursuing, I develop it in more detail. I look at how DAs interact with context (Sect. 5), at what kind of possibility claim is to be associated with a given DA (Sect. 6), and at how to accommodate the gradability of dispositional adjectives (Sect. 8). Along the way, I compare and contrast the view with views in its vicinity (Sects. 7-8).

Finally, I consider how the context-sensitivity and pragmatics of DAs allow one to deal with apparent counterexamples (Sect. 9).

\section{A warm-up argument for the view}

Let us begin by considering how DAs behave within both conjunctive claims and embedded contexts. For instance, consider the following claims:

(5) \# The glass is not breakable, but it can be broken.

(6) \# The block of marble is not fragile, but it can be broken easily.

In at least many contexts, both (5) and (6) sound infelicitous. Prima facie, they appear to involve some sort of contradiction. ${ }^{15}$

Let me give you one such context for (6):

\footnotetext{
15 Contextual shifts mid-sentence may of course allow for conversational contexts in which (5)-(6) are felicitous even if both conjuncts are possibility claims-see Sect. 5.
} 
Context for (6). There is a block of marble in front of a child and his mum, in their house (the parents are artists). The child says: "The block of marble is not fragile, but it can be broken easily!" He then tries to break the block with his bare hands.

In this context it would be appropriate to tell the child that his sentence is not a happy one, because fragile objects can be broken easily. But then here (6) is infelicitous.

One can contrast this with claims such as:

(7) If the glass were hit it would not break, but it can be broken.

(8) If the block were hit it would not break, but it can be broken easily.

These claims sound perfectly felicitous in several contexts. Here is one such context for (8):

Context for (8). The block is made of marble and really hard to break. But there is a sledge hammer at hand.

In this context, one could easily imagine someone saying: "This block is not fragile, but it can be broken easily: I'll show you with my sledge hammer!". So here (8) is felicitous.

These data suggest two things. First, they suggest that there is a semantic distance between the counterfactual claims contained in (7)-(8) and the DAs contained in (5)-(6). This supports once again the idea that DAs are not semantically close to counterfactual claims. ${ }^{16}$

Second, on the assumption that the modals 'can' and 'it is possible for' both express possibility, the infelicity of (5)-(6) suggests the following. Each of the DAs contained in, respectively, (5) and (6) implies the denial of the corresponding overt possibility claim contained in (5) and (6), respectively. For instance, the claim that the glass is fragile appears to imply that the glass can be broken easily. If so, this claim contradicts (5)'s overt possibility claim, according to which the glass cannot be broken easily. Hence, (5) sounds infelicitous. This picture provides us with a reason to think that DAs imply possibility claims.

The linguistic data we just appealed to apply cross-linguistically. I will focus on French and Spanish. Consider the sentences corresponding to the translation of (5)(6) in French and Spanish, respectively:

(5-i) \# Le verre n'est pas cassable [or: le verre est incassable], mais il peut être cassé.

\footnotetext{
${ }^{16}$ Consider also negation. If I say that this rock isn't breakable, I don't say that it doesn't necessarily break. I say that it is not possible for it to break. Similarly, if I say that this rock is not fragile, I don't say that it does not necessarily break easily; I say that it is not possible for it to break easily. On the assumption that counterfactuals express restricted necessities, these data also prove a semantic divergence between DAs and counterfactual claims.
} 
(5-ii) \# El vaso no es rompible, pero se puede romper [or, more literally: puede ser roto]. ${ }^{17}$

(6-i) \# Le bloc n'est pas fragile, mais il peut être facilement cassé.

(6-ii) \# El bloque no es frágil, pero se puede romper fácilmente.

Just like (5)-(6), each of (5-i)-(6-ii) is infelicitous in many contexts. ${ }^{18}$

Similarly, consider the following translations of (7)-(8) in French and Spanish, respectively:

(7-i) Si l'on percutait le verre, il ne se casserait pas, mais il peut être cassé.

(7-ii) Si le pegáramos, el vaso no se rompería, pero se puede romper.

(8-i) Le bloc ne se casserait pas si on le percutait, mais il peut être cassé facilement.

(8-ii) Si le pegáramos, el bloque no se rompería, pero se puede romper.

These sentences also appear felicitous in many contexts. This reinforces the suggestion that DAs are semantically distant from counterfactual claims, and that there is an implication from DAs to corresponding possibility claims.

Further embedded contexts also support the implication in the other direction, namely the implication from a possibility claim to a given DA. Consider the following claims:

(9) Since it is possible for it to be broken, this glass is breakable.

(10) Since this glass can be broken easily, it is fragile.

Each of (9)-(10) sounds felicitous in several contexts. Here is one such context for (10):

Context for (10) We are at a crystal exhibition and a mum is looking at a fine glass. Here 6 years old child is on her side. The child asks: "Why did the tourguide say that this glass is fragile?"

It seems that in this context it would be perfectly appropriate for the mum to answer her child thus: "The tour-guide said that this glass is fragile because it can break easily. Since the glass can break easily, it is fragile". But then in this context (10) is felicitous.

One way of explaining the felicity of (9)-(10) is to suggest that each of these claims relies on there being an implication. The fact that (9) is felicitous suggests that the claim that it is possible for the glass to be broken implies that the glass is breakable. The fact that (10) is felicitous suggests that the claim that the glass can be broken easily implies the claim that the glass is fragile. Nor is it is difficult to think

\footnotetext{
${ }^{17}$ I use the intransitive construction in Spanish because Spanish native speakers tell me they find themselves more inclined to use of this construction over the passive one. Note that the passive construction entails the corresponding intransitive construction here.

18 Further romance languages can be used to confirm the point in the main text. Note that the most natural translation of breakability-ascriptions in Italian involves a modal auxiliary ('it is breakable' translates: 'si puó rompere'), which also provides support for the Possibility View. Apart from this translation point, Italian data also further confirm what we get with English, French and Spanish.
} 
of further types of embedded contexts which point towards the same conclusion. ${ }^{19}$ This supports the idea that possibility claims imply DAs.

Once again, the data are not confined to the English language. The same applies for instance to the corresponding sentences in French and Spanish:

(9-i) Puisque ce verre peut être cassé, il est cassable.

(9-ii) Dado que se puede romper, este vaso es rompible.

(10-i) Puisque ce verre peut être cassé facilement, il est fragile.

(10-ii) Dado que se puede romper fácilmente, este vaso es frágil.

These sentences also appear felicitous in many contexts, and thus give us further reasons for assuming that possibility claims imply DAs.

As a result, conjunctive claims and embedded contexts support the view that there is a biconditional implication between a given DA and a corresponding possibility claim. There is then reason to think that DAs simply express possibility. No doubt, there might be other explanations of why the claims just given sound (in)felicitous. But other things being equal, the Possibility View is worth pursuing.

\section{Context-sensitivity and modal bases}

Having established that the Possibility View deserves attention, let us now develop it further. As we have seen in Sect. 2, the view relies on the idea that DAs are assessed by quantifying over a given set of possible worlds. But which worlds?

Just like for standard possibility modals—-such as 'it is possible for...', 'possibly...', and 'can'-the context determines at least in part what possible worlds we take into account for assessing a modal claim in the given context. That is, it selects salient features that need to be kept fixed in all the accessible worlds. Call the set of these worlds the modal base. ${ }^{20}$

\footnotetext{
19 Consider the following claim: 'Although this glass can be broken, it is unbreakable'. The claim sounds infelicitous. Plausibly, this is because 'this glass is unbreakable' implies 'this glass cannot be broken', which contradicts 'this glass can be broken'. A similar explanation would account for the infelicity of 'Although this glass is unbreakable, it can be broken'. One obtains similar results with claims involving 'because'.

20 I do not assume that context-dependency is always fully resolved in actual contexts (cf. Kratzer: 2013, 197-198). Formally, moreover, one can implement the context-sensitivity mechanism in different ways. The current most influential framework has been developed by Kratzer (1977, 1981, 1991, 2012, 2013) and implemented by Hacquard (2006, 2010), inter alia. It takes context to determine not only what must be kept fixed in the relevant worlds (the modal base) but also to impose an ordering on these worlds via a function (the ordering source) which arranges them in terms of closeness to a contextually determined set of propositions (the ideal). The ordering source primarily aims at dealing with comparative sentences, and has been called into question-especially (but not only) in connection with epistemic modals (Portner 2009; Yalcin 2007, 2010; Lassiter 2011, 2017, inter alia). The remarks in the present section are relevant to the individuation of the modal base. Note that dispositional adjectives do not act as epistemic modals, since the relevant modal base is circumstantial: a set of salient circumstances has to hold in each world included in the modal base.
} 
One should not assume that, for a given context, the modal base of a given DA will always be the same as the modal base of another corresponding possibility claim. Consider:

(11) The glass is fragile.

(12) The glass can be broken easily.

It is easy to conceive of conversational contexts in which the following claims can be interpreted as referring to the same glass, and are both possibility claims. Yet, they interact with context slightly differently.

To see that DAs can interact with context differently from other possibility claims, let's look at a couple of examples. Assume the following scenario:

Special Substance. There is a glass whose material breaks under no circumstances. Next to the glass there is a special substance. If one puts it on the glass, it modifies the material of the glass, and makes the glass easy to break. It is possible to put the substance on the glass.

One could truly say: "The glass can be broken easily: you just need to put the special substance on it!". Here, in order to assess whether a glass is fragile, we look at worlds which keep the actual intrinsic features of the glass fixed. But in order to assess whether the glass can be broken easily, we also look at possible worlds in which some actual properties of the glass get modified. So (11) comes out false, and (12) true.

Now assume the following instead:

Box. There is a cup made of glass in a sealed box which makes it impossible for someone to break the glass.

One could say: "Although the glass inside the box is fragile, the box makes it impossible for us to break the glass". In the given context, when assessing whether a glass is fragile one only considers possible worlds in which a glass is not in the box; whereas in assessing whether the glass can be broken one also considers worlds in which the glass is in the box. But then, (11) comes out true, and (12) false. ${ }^{21}$

Moreover, what specific restrictions we pose on the modal base varies not only from context to context, but also from one dispositional adjective to another. For some DAs, the relevant reading is often one on which the accessible worlds mostly preserve some of the intrinsic features the disposition-bearer has in the evaluation world, while allowing for maximal variation in external circumstances. For instance, claims involving 'fragile', such as

(13) Mummy's vase is fragile.

are often assessed against a modal base that keeps the structural properties of the vase fixed, and allows for a relatively maximal variation in external circumstances.

\footnotetext{
${ }^{21}$ Often the denial of DAs is simply devoted to stress the prevention of external agency. Adjectives such as 'washable', 'disposable' and 'stoppable' work just like 'breakable' in this respect.
} 
However, consider a dispositional adjective like 'visible'. One can easily imagine a touristic guide saying: "On a sunny day, the mountain is visible". In this context, whether a mountain is visible depends, among other things, on whether it is a sunny day or not. So the claim

(14) The mountain is visible.

is assessed by looking at worlds in which the atmospheric conditions are the same as they are in the world of evaluation. ${ }^{22}$ Similarly, consider:

(15) The target is vulnerable.

Whether a military target is vulnerable depends at least in part on external factors, such as the target's location. So we assess (15) by looking at worlds in which the target's location is the same as in the world of evaluation.

As a result, the context-sensitivity of DAs, just like that of modal claims in general, is extremely flexible. Different modal claims can be assessed in accordance with different modal bases within one and the same context of utterance. Moreover, the determination of the modal base depends both on the dispositional adjective in question and on further features of the context of utterance.

\section{Fine-grained manifestations}

So far, we have seen that something is F-able just in case it is possible for it to manifest that disposition. One question the Possibility View still needs to address is how exactly one should individuate the possibility associated with a given DA. In other words, we need to say more about what the manifestation of a given disposition is.

The literature on dispositions generally assumes a coarse-grained picture of manifestations. It takes manifestations to be sufficiently characterized by a verb alone. ${ }^{23}$ For instance, the manifestation of fragility is supposed to be exhaustively denoted by 'breaking', which in turn is taken to denote the event of breaking. Similarly, the manifestation of irascibility is supposed to be exhaustively characterized by 'getting angry', which in turn denotes the event of getting angry.

To my knowledge, the coarse-grained picture of manifestations goes undefended in the current debate. Nor does it have a long tradition. Aristotle, for instance, thought that the property of being soft is a disposition (in the contemporary sense of the term), and that the manifestation of this disposition is being cut easily (Categories, 9a27-9). On this alternative picture, the manifestation of the disposition is being cut in an easy way, and its characterization includes a qualification of the verb, namely the qualification 'easily'.

\footnotetext{
22 Accepting that, say, 'visible' is a dispositional adjective does not by itself settle whether one should admit of extrinsic dispositions in one's metaphysics, and how to account for them. For an account of extrinsic dispositions, see McKitrick (2003); for skepticism, see Park (2017).

23 Cf. Mackie (1977), Lewis (1997a), Manley and Wassermann (2007, 2008) and Vetter (2014, 2015), inter alia.
} 
Let us generalize this thought. Call a fine-grained picture of manifestations a picture on which the manifestation of a disposition is in some cases best individuated by means of expressions that include qualifications of the verb, such as for instance adverbial qualifications. For instance, the manifestation of fragility is being broken easily; the manifestation of breakability, on the other hand, is successfully characterized merely by 'being broken'. So manifestations can be events which are more fine-grained than it is usually assumed. I shall now argue that the correct account of DAs has to assume the fine-grained picture of manifestations, and the coarse-grained picture is to be dismissed.

First, consider again the argument from embedded contexts given in Sect. 4. Among other things, the argument gives us a reason to assume that claims such as the ones below, at least in some contexts, imply one another:

(16) $N$ is fragile.

(17) $N$ can be broken easily.

This suggests that the manifestation of fragility should be characterized with a verbal expression in its passive form. ${ }^{24}$ It also begins to provide us with reason for thinking that the qualification 'easily' is relevant to the characterization of the manifestation of fragility.

Now let us take a closer look at (17). This claim actually contains two verbal expressions: the modal 'can' and the expression that falls within the scope of the modal, 'be broken'. Can 'easily' modify either of them? Some authors suggest that 'easily' can modify modals. ${ }^{25}$ I think there might be ground for skepticism here, especially in connection with circumstantial modality. But for present purposes I focus on making a narrower claim. My claim is this: (16) and (17) imply each other in many contexts, provided we take 'easily' to modify 'being broken' .

Let me give some general reasons for accepting that 'easily' can modify verbs that fall within the scope of a modal. I do not expect this point to be controversial. But given that it is crucial for my purposes, it is worth giving it explicit support. Consider how 'easily' coordinates with other adverbs that cannot modify a modal, such as 'quickly':

(18) The glass can be broken quickly and easily.

Coordination typically only works when you are coordinating things of the same type. (18) is felicitous, so in this case coordination works. This suggests that here both adverbs modify the same item, namely 'be broken', and thus 'easily' acts as a manner adverb here.

\footnotetext{
${ }^{24}$ Similarly, the manifestation of visibility is something like being seen; the manifestation of thinkability is something like being thought; etc.

25 Vetter (2014, 2015: 70ff) suggests that there is a bi-implication between (16) and (17) when 'easily' is interpreted as qualifying the modal 'can'. I discuss her resulting semantics in Sect. 7, and show how my account avoids the difficulties of Vetter's in Sect. 8. For another account of how 'easily' may qualify modals, see Klecha (2014a, b). Klecha (2012, 2014b: chapter 5) argues against the view that 'possible' is gradable also within epistemic contexts - contra Lassiter (2011), inter alia-but claims it can nonetheless be qualified by 'easily'.
} 
Now consider this exchange:

"That glass was fragile."

"Why?"

"It broke easily."

Here 'easily' does not modify the modal-there is no modal for it to modify! This gives us reason to assume that (16) correlates with (17) when the later interpreted in such a way that 'easily' modifies 'break'. But then, we have a reason to conclude that 'being broken' is not a correct characterization of the manifestation of fragility. To characterize this manifestation correctly, we need a more specific expression, such as 'being broken easily' — where 'easily' gets precisified by context and often means, roughly, 'under little impact force' (cf. Sect. 8).

The point extends to other dispositional adjectives. Consider:

(19) The task is feasible.

Roughly, something is feasible just in case it can be accomplished with an acceptable amount of effort. So the manifestation of feasibility is something like: being accomplished with an acceptable amount of effort. More generally, the manifestation of a disposition is often best characterized not merely by means of a verb, but a verb together with some qualification. The resulting expression picks up a more specific event than the event denoted by the verb alone. So some dispositions have fine-grained manifestations.

Note that the fine-grained picture of manifestations indirectly supports the Possibility View. The coarse-grained picture does not fit well together with an immediate implication of the view, namely the sufficiency of manifestations thesis - the thesis that actually manifesting a disposition is sufficient for possessing it (cf. Sect. 2.1). When joined with a coarse-grained picture of manifestations, the sufficiency of manifestations thesis yields bad results. For instance, on the assumption that breaking is the manifestation of fragility, the sufficiency of manifestations implies that if something breaks, it is fragile. Yet a bull-dozer's breaking that a block of marble doesn't seem to warrant the conclusion that the block is fragile. ${ }^{26}$ So one should not endorse the sufficiency of manifestations thesis and a coarse-grained picture of manifestations together.

Now, when joined together with the sufficiency of manifestations thesis, the finegrained picture of manifestations yields good results. For instance, it tells us that, at least in many contexts, if something gets broken easily, then it is fragile-as seems plausible. Since the fine-grained picture of manifestations is independently motivated, this further supports the Possibility View itself. ${ }^{27}$

\footnotetext{
${ }^{26}$ Similarly, getting angry is taken to be the manifestation of irascibility. But the fact that Gandhi got angry once (let's suppose) does not seem to show that he was irascible.

27 Note also that since the Possibility View assumes a fine-grained account of manifestations, it can explain why fragility ascriptions, say, imply breakability ascriptions but not vice versa. Breakability and fragility have different manifestations: the manifestation of breakability is the event of being broken, and the manifestation of fragility is the event of being broken easily. On the assumption that getting broken
} 


\section{Comparison with (some) alternative views}

Having stated the central claims of the Possibility View, it can be useful to compare and contrast it with other views that do not attempt at reducing disposition ascriptions to DAs. In particular, there are two main alternatives to the standard counterfactual picture that lie somewhat in the vicinity of the Possibility View: Manley and Wassermann $(2007,2008)$ 's, and Vetter $(2014,2015)$ 's. This section looks at each of these views, and clarifies where and why the Possibility View differs.

\subsection{Manley and Wassermann (2008)}

Manley and Wassermann (2008) offer an account of expressions of the form ' $x$ is disposed to $\varphi$ ' while taking some distance from counterfactual accounts:

(MW) ' $x$ is disposed to $\varphi$ ' is true iff $x$ would $\varphi$ in some suitable proportion of C-cases.

where a C-case is a sufficiently specific possible scenario, and the context determines the threshold for suitability-namely, in how many scenarios $x$ has to manifest the disposition in order to possess that disposition. For instance, an object is disposed to break just in case it breaks in enough possible scenarios.

We have seen that expressions of the form ' $x$ is disposed to $\varphi$ ' fall beyond the scope of this paper. But one can extend Manley and Wasserman's view to DAs in the following way:

(MW') ' $x$ is F-able' is true iff $x$ would $\varphi$ in some suitable proportion of C-cases.

Here ' $\mathrm{C}$ ' picks up the stimulus-conditions for the manifestation of a disposition. For instance, in the case of fragility $\mathrm{C}$-cases are cases in which $n$ is stressed. Moreover, the suitable proportion of C-cases is established by context.

The Possibility View differs from (MW') in a number of ways. First, (MW') assumes that, at least for many dispositions, it is possible to specify a clear and extrinsic stimulus conditions that trigger the manifestation of a given disposition. For instance, fragility requires referring to stress conditions of a given sort. ${ }^{28}$ The Possibility View does not assume that there are extrinsic conditions for the manifestation of a disposition that can be independently specified.

Second, (MW') claims that the modal force of a DA varies with context-the context allegedly tells us in how many possible worlds a manifestation has to occur for the DA to be true. Among other things, this threatens to make a DA ambiguous - in some contexts it may stand for a necessity claim, in others for a

Footnote 27 continued

easily is a way of getting broken, the fact that it is possible to break easily implies that it is possible to break (and not vice versa). But then, if something is fragile, it is breakable (and not vice versa).

${ }^{28}$ For objections to this assumption, see Vetter $(2011,2014,2015)$. 
possibility clams, and yet in others for something in between. ${ }^{29}$ By contrast, the Possibility View endorses a more straightforwardly unitary account of DAs, since it takes all these claim to be equivalent to possibility claims.

Third, (MW') so far lacks a necessary ingredient: it does not tell us how worlds are to be counted. Yet it appeals to a kind of quantification that requires for something to hold in a relevant proportion of accessible worlds. Thus to fully complete (MW') a criterion for counting worlds would need to be provided. The Possibility View, on the other hand, does not need to provide such a criterion.

Finally, Manley and Wasserman assume the coarse-grained picture of manifestations. They preserve for instance the standard assumption that the manifestation of fragility is sufficiently described by the associated verb, and thus amounts to an event of breaking. Accordingly, they deny the sufficiency of manifestations thesis. By contrast, the Possibility View opts for a fine-grained picture of manifestations and endorses the sufficiency of manifestations thesis (cf. Sects. 2.1 and 6). We have already given reasons for preferring a fine-grained picture of manifestations (Sect. 6). We shall also see later that the endorsement of a coarse-grained picture of manifestations can lead to a problematic account of the gradability of dispositional adjectives (Sect. 8.1).

\subsection{Vetter $(2014,2015)$}

Vetter (2014, 2015) posits two classes of DAs. DAs such as 'the glass is breakable', she says, are possibility claims-just like the Possibility View says. Yet other DAs, such as 'the vase is fragile' and 'Socrates is irascible', have a stronger modal force:

$x$ is fragile simpliciter just in case it breaks in a sufficiently large proportion of the relevant possible worlds, where context may determine what counts as sufficiently large. (2015: 73)

That is:

(V) ' $x$ is fragile' is true iff $x$ breaks in a few possible worlds.

Here context determines both the domain of quantification and the modal force denoted by 'a few' - in at least how many worlds $a$ has to break to count as fragile.

Now (V) is remarkably close to (MW'). One difference is that for Vetter the relevant threshold is always relatively close to-but not identical with-the threshold of possibility, rather than necessity. So on her account the number of

\footnotetext{
29 To avoid ambiguity, one may either (i) try and develop this view by suggesting that the modal force of dispositional adjectives is a free variable to be saturated by context, or (ii) embrace polysemy. On (i), the interaction with context of these modals works like that of 'that': they have a different content in different contexts, but somehow the same sort of character. I know of no such developed account, and I am skeptical about its prospects. The main challenge for such a view would be to give a plausible story about how to distinguish character and context for modals. On (ii), a dispositional adjective becomes a single lexical item with many related senses. This amounts to accepting that many dispositional adjectives involve a weak form of ambiguity. Viebahn and Vetter (2016) propose polysemy for 'may' and 'can'.
} 
worlds in which something has to break to count as fragile is always relatively low. ${ }^{30}$

The Possibility View differs from Vetter's in three main ways. First, Vetter does not offer a fully unified account of DAs. She posits two classes of DA, and adds that the second class of DAs is not equivalent to standard possibility claims - contrary to what the Possibility View says. Just like (MW'), then, this view threatens to posit an ambiguity amongst DAs, in that it claims that the modal force of the second set of DAs is ultimately context-dependent. ${ }^{31}$

Second, Vetter's understanding of manifestations sides yet again with the standard assumption that these manifestations are best characterized merely by a verb, and accepts that the manifestation of a disposition of fragility just is the eventtype of breaking. As we have seen, however, a fine-grained account of manifestation is ultimately preferable (Sect. 6).

Third, Vetter's view requires certain conditions to hold in a large proportion of relevant possible worlds. To be complete the view would have to tell us how exactly the worlds are to be counted, both in finite and infinite domains. On this view, when assessing DAs such as fragility claims we have to conclude that the claim is true in case the object breaks in a sufficiently large proportion of relevant worlds. Even accepting that context has a role to play here, one cannot apply the view without criterion for counting worlds. By contrast, the Possibility View simply appeals to existential quantification over possible worlds.

To sum up, the Possibility View offers a more unified and straightforward account of DAs than its closer alternatives. It also endorses a different and independently motivated picture of manifestations. Moreover, in the next section I show that the differences between the Possibility View and these alternatives allow my view to better account for the gradability of dispositional adjectives.

\section{The gradability of dispositional adjectives}

Gradability is the linguistic phenomenon whereby adjectives can be naturally modified by qualitative modifiers such as 'more', 'less' and 'very', and appear in comparative clauses. Thus 'green' is a gradable adjective, since it can appear in comparative clauses, as in:

(20) This leaf is greener than that one.

Several dispositional adjectives are gradable too. They can appear in comparative clauses, as in:

(21) This vase is more fragile than that one.

(22) I am more irascible than you are.

\footnotetext{
30 Vetter (2015: 73ff).

31 One may try to avoid ambiguity by pursuing either polysemy or indexicality. Since Vetter accepts that 'can' and 'may' involve polysemy — in Viebahn and Vetter (2016)—she would probably extend polysemy to dispositional adjectives.
} 
They can also be qualified with 'very', 'extremely', 'incredibly', etc.

Gradability is hotly debated amongst linguists at the moment. What tends to be accepted is that accounting for the gradability of property ascribing adjectives requires one to specify an ordering. Along that ordering, one calculates the degree to which an entity has the property, and how the degree gets fixed (cf. Klein: 1980, 1991; Kennedy and McNally 2005). For example, one can account for

(23) Tom is taller than Jim,

by specifying the degree of Tom's and Jim's tallness along the ordering of height. If on that ordering Tom's degree of tallness is higher than Jim's, (23) is true. ${ }^{32}$

The individuation of an ordering appears to be difficult for DAs, however. What is the relevant ordering for 'fragile', for example? This seems unclear. Some views about the gradability of dispositional adjectives have appeared within the dispositions debate (Manley and Wassermann 2007, 2008; Vetter 2014, 2015). In what follows, I argue that these accounts are problematic (Sect. 8.1). Then I show that, given the Possibility View, the account of the gradability of non-dispositional adjectives can be extended to dispositional adjectives in a straightforward way (Sect. 8.2).

\subsection{Current accounts}

The most developed accounts of the gradability of dispositional adjectives have been put forward precisely by Manley and Wassermann $(2007,2008)$ and Vetter (2014, 2015), the authors who put forward the closest alternatives to the Possibility View. As far as gradability goes, their two accounts are remarkably alike.

Manley and Wassermann suggest:

$x$ is more disposed to $\mathrm{M}$ when $\mathrm{C}$ than $y$ iff $x$ would $\mathrm{M}$ in more $\mathrm{C}$-cases than y. (2007: 72ff; 2008: 17)

For instance, $a$ is more fragile than $b$ just in case $a$ breaks when struck in more $\mathrm{C}$-cases than $b$, where $\mathrm{C}$-cases are relevant possible scenarios selected by context. Similarly, Vetter writes:

$x$ is more fragile than $y$ just in case $x$ breaks in more of the relevant worlds than $y$. (2015: 73)

So: $x$ is more fragile than $y$ just in case there are more scenarios in which $x$ breaks than scenarios in which $y$ breaks.

Vetter gives us more details about how to think about possible scenarios than Manley and Wasserman. I will focus on discussing her version of this account of gradability. She suggests:

\footnotetext{
32 Scholars often talk about a scale, rather than an ordering (cf. Klein 1980, 1991; Kennedy and McNally 2005, 2010, inter alia). But strictly speaking to get gradability one only needs an ordering, and not a metric. Plausibly, some adjectives should be associated merely with an ordering rather than a metric, since not for every gradable adjective $\mathrm{F}$ one can say things like ' $a$ is twice as $\mathrm{F}$ as $b$ '. In the main text, I talk about orderings, and assume that scales are special cases thereof.
} 
To get the proportions right, we should quantify not over worlds but over cases or centered worlds: triples of a world, a time, and an object. An irascible person will typically get angry any number of times within one world, and a transmissible disease will typically be transmitted more than once. What should determine the proportion that makes a person count as irascible, or a disease as transmissible, is not the number of worlds in which the person becomes angry at least once or the disease transmitted at least once, but rather the number of individual instances of anger or transmission: in other words, of cases (2015: 77).

The idea is simple enough: $x$ is more fragile than $y$ just in case, for a given context of utterance, $x$ breaks in more centered worlds than $y$ does. In general, for a dispositional adjective 'F-able', $x$ is more F-able than $y$ just in case $x$-es in more centered worlds than $y$.

Yet, I submit that this account of gradability is problematic. Assume the following scenario:

$R$-bulls and $G$-bulls. R-Bulls get angry when they see something green. They also get angry when they see something red. G-bulls behave just like r-bulls under all circumstances, except when they see something green; the sight of green instantly kills them. ${ }^{33}$

Now consider the claim

(24) R-bulls are as irascible as g-bulls.

Given our scenario, Vetter's account predicts that (24) is false. There are more centered worlds in which r-bulls get angry than centered worlds in which g-bulls get angry. R-bulls get angry in the centered worlds in which they only see something green. But g-bulls cannot survive in these centered worlds. Hence, r-bulls are more irascible than g-bulls.

Yet, strictly speaking, the issue of whether they are as irascible as r-bulls seems to be open. The question of whether g-bulls are as irascible as r-bulls is, at the very least, intelligible and not trivial. One could imagine a group of biologists who takes up this research question, and tries to find out whether g-bulls are more irascible than r-bulls. So the truth of (24) should not be rejected out of hand by our semantics - contrary to what the account says.

On the face of this difficulty, one might try to fix Vetter's account in three main ways. First, one could suggest that likelihoods are to be evaluated conditionally. Given $R$-bulls and $G$-bulls, one has to assess (24) by considering the probability of getting angry conditional on seeing, say, something red. The probability of an r-bull getting angry on seeing something red is high. By definition, the probability of a g-bull getting angry on seeing something red is also high. Accordingly, r-bulls turn out to be as irascible as g-bulls.

One can understand the resulting view as saying that DAs are equivalent to conditionals with probabilistic truth-conditions. On this view, g-bulls are irascible

\footnotetext{
33 The set-up is in many ways analogous to Kripke's killer-yellow scenario—cf. Lewis (1997b: 344).
} 
just in case if they see something red, they get angry. This conditional is true just in case, given the probability that g-bulls see something red, the probability that g-bulls get angry is sufficiently high. But Vetter herself points out that conditionalizing on triggering conditions yields unsatisfactory accounts of DAs (cf. Vetter 2011, 2014, 2015: Sect. 2.2-2.6). Given that one wants to specify the general ascription conditions for irascibility, it is implausible to suggest that something is irascible just in case, if it sees something red, it gets angry. Surely, redness is just one of many triggering conditions of irascibility. Nor can one say: something is irascible just in case, if something makes it angry, it gets angry. For this is trivial.

Alternatively, one may try to posit constraints on the domain of possible worlds which are relevant for assessing a given DA. Perhaps, for every world in the domain of quantification, the context keeps fixed the properties of an object which entail the possession of the disposition, and allows for maximal variation otherwise. Thus: $x$ is F-able just in case, in a few of the worlds in which $a$ has a set of properties which entail the possession of F-ability, $x$ is F-able. Yet, the resulting account makes (24) true of necessity. For the account is trivial. Everything is such as to have a given disposition in any world in which it has a set of properties which entail the possession of the disposition.

Third, one might stipulate that the context allows us to keep (24) open. We thus get: $x$ is F-able just in case in a few of the worlds in which $x$ preserves a given set of properties, $x \varphi$-es, where the relevant set of properties is determined by context in such a way that one can predict the truth-value of (24) correctly.

As it stands, however, this view is ad hoc. It tells us that DAs interact with context in a way which makes the truth-value of claims such as (24) come out right, without giving us independent reasons to think that the context interacts with DAs in the relevant way, nor further details about how the interaction with context is supposed to go. It is unclear that any such account is available, and that it would predict the needed results. In its absence, Vetter's view has not been shown to correctly capture the gradability of dispositional adjectives.

\subsection{My account: gradable manifestations}

Let us take stock. So far, we have seen that a challenge for a view about DAs is to offer an account of the gradability of dispositional adjectives. We have also seen that the main account of their gradability in the literature are problematic. I will now put forward an alternative account of the gradability of dispositional adjectives.

Other things being equal, it is desirable to capture the gradability of all gradable adjectives in a unified way. Accordingly, it is worth aiming at extending the framework already in use to account for adjectives like 'tall' and 'green' to dispositional adjectives too. As we have seen, on these accounts gradability specifies the degree to which an entity possesses a given property is measured against a contextually determined ordering. To extend such an account to dispositional adjectives, one needs to explain how to individuate their orderings.

Interestingly enough, the characterization of manifestations endorsed by the Possibility View allows one to individuate an ordering for dispositional adjectives in 
a straightforward way. We now know that DAs like fragility ascriptions require us to include some qualification of the verb used to characterize the manifestation of a given disposition. Say, something is fragile just in case it is possible for it to be broken easily (cf. Sect. 6).

Now the qualifications of the verb that are part of the characterization of the manifestation of certain dispositions can be gradable. For instance, 'easily' is gradable: one can do things more or less easily, say. This allows us to determine the ordering in play in comparison clauses involving dispositional adjectives. For instance, in a context in which 'easily' means the same as 'with little impact force', $x$ is more fragile than $y$ just in case $x$ can break with less impact force than $y{ }^{34}$

Similarly, consider 'feasible'. Its manifestation contains some qualification something like 'with little effort', where what counts as little effort is a contextsensitive matter. This allows us to determine the ordering in play in comparison clauses involving feasibility: $x$ is more feasible than $y$ just in case $x$ can be accomplished with less effort than $y$.

Generally put, qualifications on the manifestation-verb account for the fact that dispositional adjectives are gradable. This is because the gradability of a dispositional adjective depends on the gradability of the manifestation associated with the relevant disposition. The more an object can break easily, the more fragile it is. The more a task may can get accomplished with little effort, the more feasible it is. The degree to which something possesses a disposition depends on the degree to which it manifests the disposition in the relevant manifestation-world(s). ${ }^{35}$ In a slogan: dispositions are gradable because they have gradable manifestations. So one can measure the degree to which something has a disposition in term of the degree to which it manifests the disposition in the manifestation-world(s). ${ }^{36}$

Now consider a claim of the form:

(27) $x$ is more fragile than $y$.

Given what we have just seen, the claim is true just in case $x$ manifests fragility more than $y$. Manifesting fragility amounts to breaking easily, where what qualifies as to breaking easily is context-sensitive. Suppose we are in a context where

\footnotetext{
34 This explains why it is more natural to grade a dispositional adjective like 'fragile' than an adjective like 'breakable'. When 'breakable' also appears in gradable constructions, this is because we grade breakability by using an ordering that relies on features of the manifestation, being broken.

35 The account can thus be developed further by building on current accounts of how to measure and compare events - see Wellwood et al. (2012) for an overview. See also Wellwood (2015) for an account of gradable predicates that maps them to degrees of events or states.

${ }^{36}$ Formally, one can regiment this picture in different ways. One may try to appeal to Kratzer's ordering source (introduced in Kratzer 1981, 1991, 2012). If so, the gradability of claims such as ' $x$ is more likely to break than $y$ ' might be accounted for in terms of degrees of closeness to an ideal set by contextroughly, ' $x$ is more likely to break than $y$ ' just in case at least one possible world in which $x$ breaks easily is closer to the ideal than any world in which $y$ breaks easily. However, note that the ideal would also have to encode that breaking under less impact force leads to a higher ranking (for an account of modal comparisons that encodes some higher-order information of this sort in the ideal, see Katz et al. 2012; Silk 2017). Alternatively, one might order words without appealing to an ideal. What one needs here is a contextually-determined standard of comparison that gives us an ordering of the relevant possible worlds, and a function that tells us how to establish whether $x$ has a given disposition to a higher degree than $y$.
} 
breaking easily means to break under an impact force that is equal to or lower than $2 \mathrm{~N}$. I suggest that in this context (27) is true just in case there is a relevant possible world in which $x$ breaks under less impact force than $y$ breaks in any of the relevant possible worlds.

We are now in a position to re-consider the objection I put forward against Vetter's account, the R-bulls and G-bulls case. As we have seen, this objection brings out how Vetter does not properly account for the gradability of 'irascible'. Her view wrongly rules out that g-bulls and r-bulls are equally irascible. Does my account do better?

I submit that it does. On the Possibility View, as we have just seen, the gradability of the manifestation of a disposition transfers to the disposition itself. Thus we can account for the r-bulls and g-bulls case as follows: a g-bull is more irascible than an r-bull just in case it can get angry with a weaker provocation than an r-bull. Here what counts as a weak provocation is a context-sensitive matter. This account does not deny out of hand that g-bulls and r-bulls are equally irascible. Moreover, on the account it is likely to turn out to be true, for many contexts, that g-bulls and r-bulls are equally irascible. This seems to be the correct result.

\section{Two possible objections}

\subsection{Soft spots}

Objection. One may take cases like the following to be a problem for my account:

Soft Spot. A concrete block withstands heavy forces. Yet, it has one soft spot. At a particular corner, it is very easy to break. There, little force will do. ${ }^{37}$

In this scenario,

(28) The block is not fragile.

seems true. Yet, the block could be broken easily at the corner. So, on the Possibility View, (28) comes out false. ${ }^{38}$

Reply. The objection is too quick. The following claim

(29) I can walk through the wall.

seems false. Yet, quantum mechanics tells us it is physically possible for me to walk through a wall, under ordinary conditions. The probability that this happens is incredibly low. Nonetheless, it is a possible event.

News from quantum mechanics could be taken as a reason for denying that (30) is ever false. Or, more plausibly, one can assume that when we assessing (30) we usually do not consider worlds in which I walk through a wall. There are in fact

\footnotetext{
37 This case is similar to one given by Manley and Wassermann (2008: 67).

38 Vetter (2015: 10) takes this case to support her account of DAs-but see Sects. 7.2 and 8.1.
} 
contexts in which we do take this sort of world into account. For instance: "I thought I could not go through the wall; but quantum mechanics taught me that this is possible, after all!". Here, the worlds in which the speaker goes through the wall are accessible.

Something similar happens with Soft Spot. Typically, when we assess the claim that the block is not fragile, (29), we ignore the scenario in which the block gets hit at the relevant corner. Accordingly, we conclude that the block cannot get broken with little force, and take the block not to be fragile. There are, however, contexts in which we do not ignore this scenario. Consider a speaker who says: "You know what? I found out that this block is fragile: it can be broken very easily on that corner!". Plausibly, in this conversation the speaker considers the scenario in which the block gets broken easily. So she concludes that the block is fragile after all. This judgment seems very natural. This creates at least a presumption that, in some contexts, our block is correctly described as fragile.

Consider also the following scenario:

Softer Spot. The block breaks under exactly the same conditions stated in Soft Spot: it breaks only if hit in a specific way in the right bottom corner. Yet it is situated among people who very often hit blocks in their right-bottom corner in the specific way that makes our block break. It's natural for these people to hit there, with that little force.

Given Softer Spot, (29) seems false. Yet, between Soft Spot and Softer Spot nothing has changed in the breaking-conditions of the block. The block breaks easily at one and only one point. What has changed is how likely it is that, in the given context, the relevant breaking conditions hold. When we assess (29) against Soft Spot, we tend to ignore far-fetched scenarios, such as the one in which the block gets broken easily. But given Softer Spot, such a scenario is no more far-fetched.

I conclude that Soft Spot is not an objection to the Possibility View. It can be dealt with by appealing to the context-sensitivity of DAs. Nonetheless, the case is interesting in its own right. It raises questions about why we ignore certain possibilities when assessing modal claims in general, an issue which extends to necessity, counterfactual, and overt possibility claims, and deserves to be addressed in a separate occasion. ${ }^{39}$

\footnotetext{
39 Two suggestions. (i) We often seem to ignore events which have a low probability to occur. The probability that, in ordinary conditions, I walk through the wall is incredibly low. So usually in assessing 'I can walk through the wall' we ignore worlds in which this happens. Similarly, the probability that a concrete block with a weak spot gets broken with little force is low. So in assessing the claim 'the block is not fragile' we tend to ignore the only world in which the block gets broken in this way. (ii) Normality seems often set not simply by objective frequency but also by practical considerations. We tend to describe plates as fragile when doing things like moving them around by hand in the house (as distinct from bombarding them with electrons in a lab). Lewis (2016) argues that considerations of this kind also apply to the mechanism of context-sensitivity of counterfactual claims.
} 


\subsection{Payable, honourable, despicable}

Objection. Think of 'payable', 'honourable', and 'despicable'. Their modal flavour is typically deontic: they involve the modality what ought or is allowed to be the case, rather than the circumstantial modality of what can or must be the case given the circumstances. ${ }^{40}$ Worryingly, their modal force seems to be that of necessity: to say that a tax is payable appears equivalent to saying that the tax has to be paid; similarly, to describe a person as honourable sounds like to say that the person has to be honoured; and a despicable act seems to be one that ought to be despised. Accordingly,

(30) This tax is payable

(31) Your father is an honourable person

(32) The act is despicable

appear equivalent to necessity claims, and not to possibility claims-pace the Possibility View.

Reply. I agree that the perceived modal strength of the dispositional adjectives involved in (30)-(32) is often that of necessity, but disagree that (30)-(32) are counterexamples to the Possibility View. Rather, I suggest, the perceived modal strength is due to an implicature.

Although this is not the place to fully develop a technical account of the pragmatics of DAs, the basic idea is not difficult to elucidate. For claims like (30)(32), I suggest, relevance can force us to ignore the possible scenarios in which the disposition is not manifested. ${ }^{41}$ Consider (30), for example. Strictly speaking, a payable tax is an unpaid tax which can be paid by us. Yet relevance usually makes us ignore scenarios in which, say, we do not pay taxes. As a result, we interpret (30) as conveying a necessity. ${ }^{42}$

Similarly, (31)-(32) strictly speaking tell us, respectively, that your father can be (justifiably) honoured, and a given act can be (justifiably) despised. Yet relevance can make us ignore, respectively, scenarios in which we don't despise a despicable act, and fail to praise the praiseworthy act. As a result, pragmatics leads us to

\footnotetext{
40 Just like other modals, some dispositional adjectives can preferably associate with modal flavours, and which modal flavour this is varies among them. This is analogous to the fact that the modal 'ought' goes with a deontic modal force (expressing what is necessarily the case given certain norms), for instance.

41 Bassi and Bar-Lev (forthcoming) defend a similar suggestion for conditionals - that when 'If p, q' and 'If p, might q' are perceived as equivalent, the perceived strength of the former conditional is due to an implicature. I suspend judgement on whether Bassi and Bar-Lev's account of conditional claims is correct, but share their assumption that implicatures can lead us to interpret a claim involving existential quantification as involving universal quantification instead.

42 It is worth drawing a connection with overt possibility claims: 'I might pass on the wine' can be a polite way of saying 'I will pass on the wine', and 'You may pay for parking up front' a polite way of saying 'You must pay for parking up front'. This strengthening is clearly pragmatic in nature. Plausibly, DAs undergo analogous phenomena. (Thank you to Matthew Mendelkern for the example.).
} 
interpret (31)-(32) as necessity claims. This is not a problem for the Possibility View. On the contrary, the view has the merit of being able to give a unified account of DAs, while also explaining why some DAs can apparently involve a different modal force.

\section{Conclusion}

I conclude that DAs express possibility, as the Possibility View says. This view is supported by embedded contexts. Moreover, since the manifestations of dispositions can be graded, the view can account for the gradability of dispositional adjectives. Furthermore, apparent counterexamples to the Possibility View can be explained away by taking into account the context-sensitivity and pragmatics of DAs.

It will be interesting to see what implications this account has for the metaphysics of dispositions. It will also be interesting to investigate whether expressions such as generics (e.g. I jog, I smoke) and other property ascriptions (e.g. the car is fast) also express possibility. ${ }^{43}$ But this is for another occasion.

Acknowledgements Thank you to all the wonderful people I had the pleasure of discussing this topic with, including Zack Al-Witri, Cian Dorr, Dorothy Edgington, Daniel Hoek, Angelika Kratzer, Karen Lewis, Matthew Mendelkern, Eliot Michaelson, Ethan Nowak, Carlotta Pavese, Zee Perry, Jim Pryor, Alberto Tassoni, Barbara Vetter, and Timothy Williamson. I am also grateful to an anonymous referee.

Open Access This article is distributed under the terms of the Creative Commons Attribution 4.0 International License (http://creativecommons.org/licenses/by/4.0/), which permits unrestricted use, distribution, and reproduction in any medium, provided you give appropriate credit to the original author(s) and the source, provide a link to the Creative Commons license, and indicate if changes were made.

\section{References}

Aimar, S. (ms). Dispositions.

Bassi, I., \& Bar-Lev, M. (forthcoming). A unified existential semantics for bare conditionals. Sinn und Bedeutung 21.

Bird, A. (1998). Dispositions and antidotes. The Philosophical Quarterly, 48, 227-234.

Bonevac, D., Dever, J., \& Sosa, D. (2011). The counterexample fallacy. Mind, 120, 1143-1158.

Borghini, A., \& Williams, N. (2008). A dispositional theory of possibility. Dialectica, 62, 21-41.

Choi, S. (2006). The simple vs. reformed conditional analysis of dispositions. Synthese, 148, 369-379.

Contessa, G. (2013). Dispositions and interferences. Philosophical Studies, 165, 401-419.

Contessa, G. (2016). Dispositions and tricks. Erkenntnis, 81, 587-596.

Fara, M. (2005). Dispositions and habituals. Noûs, 39, 43-82.

Goodman, N. (1954). Fact, fiction and forecast. Cambridge: Harvard University Press.

Hacquard, V. (2006) Aspects of modality. PhD thesis, Cambridge, MA: MIT.

Hacquard, V. (2010). On the event relativity of modal auxiliaries. Natural Language Semantic, 18, 79-114.

Katz, G., Portner, P., \& Rubinstein, A. (2012). Ordering combination for modal comparison. Semantics and Linguistics Theory, 22, 488-507.

\footnotetext{
$\overline{43}$ For a beautiful start in this direction, see Menéndez-Benito (2013).
} 
Kennedy, C. (1995). Comparatives, indices and scope. In Sixth annual meeting of the formal Linguistics Society of Midamerica, $126 \mathrm{ff}$.

Kennedy, C. (2007). Vagueness and grammar: The semantics of relative and absolute gradable adjectives. Linguistics and Philosophy, 30, 1-45.

Kennedy, C., \& McNally, L. (2005). Scale structure, degree modification and the semantics of gradable predicates. Language, 81, 381-393.

Kennedy, C., \& McNally, L. (2010). Color, context and compositionality. Synthese, 17, 79-88.

Klecha, P. (2012). Positive and conditional semantics for gradable modals. In Proceedings of sinn und bedeutung (Vol. 16, pp. 363-376).

Klecha, P. (2014a). Modifying modality. In J. Iyer \& L. Kusmer (eds.), Proceedings of NELS, pp. 220-232.

Klecha, P. (2014b). Bridging the divide: Scalarity and modality. PhD thesis, University of Chicago.

Klein, E. (1980). A semantics for positive and comparative adjectives. Linguistics and Philosophy, 4, $1-45$.

Klein, E. (1991). Comparatives. In A. von Stechow \& D. Wunderlich (Eds.), Semantics: An international handbook of contemporary research. Berlin: Walter de Gruyter.

Kratzer, A. (1977). What must and can and must mean. Linguistics and Philosophy, 1, 337-355 [Slightly revised in Kratzer (2012, ch.1)].

Kratzer, A. (1981). The notional category of modality. In: H.-J. Eikmeyer \& H. Rieser (eds.), Words, worlds, and contexts. New approaches in word semantics. Berlin: Mouton de Gruyter, pp. 38-74 [Expanded in Kratzer (2012, ch. 2)].

Kratzer, A. (1991). Modality. In A. von Stechow \& D. Wunderlich (Eds.), Semantik: Ein internationales Handbuch zeitgenössischer Forschung (pp. 639-650). Berlin: Mouton de Gruyter.

Kratzer, A. (2007). Situations in natural language semantics. In N. E. Zalta (Ed.), Stanford encyclopedia of philosophy. Stanford: Metaphysis Research Lab, CSLI, Stanford University.

Kratzer, A. (2012). Modals and conditionals: New and revised perspectives. Oxford: Oxford University Press.

Kratzer, A. (2013). Modality for the 21st century. In J. Moeschler \& F. Raboul (Eds.), The languagecognition interface. Geneva: Librairie Doz.

Lassiter, D. (2011). Measurement and modality: The scalar basis of modal semantics. PhD dissertation, NYU Linguistics.

Lassiter, D. (2017). Graded modality: Qualitative and quantitative perspectives. Oxford: Oxford University Press.

Lewis, D. (1997a). Finkish dispositions. The Philosophical Quarterly, 47, 143-158.

Lewis, D. (1997b). Naming the colors. The Australian Journal of Philosophy, 75, 325-342.

Lewis, K. S. (2016). Elusive counterfactuals. Noûs, 50, 286-313.

Mackie, J. L. (1972). Truth, Probability, and Paradox. Oxford: Oxford University Press.

Mackie, J. L. (1977). Dispositions, grounds and causes. Synthese, 34, 361-369.

Maier, J. (forthcoming). Modal predicates. Linguistics and Philosophy.

Manley, D., \& Wassermann, R. (2007). A gradable approach to dispositions. The Philosophical Quarterly, 58, 68-75.

Manley, D., \& Wassermann, R. (2008). On linking dispositions with conditionals. Mind, 117, 59-84.

Martin, C. B. (1994). Dispositions and conditionals. The Philosophical Quarterly, 44, 1-8.

Martin, F., Pitteroff, M., \& Pross, T. (Eds.). (2016). Morphological, syntactic and semantic aspects of dispositions. Stuttgart: Online Publikations verbund der Universität Stuttgart.

McKitrick, J. (2003). A case for extrinsic dispositions. Australasian Journal of Philosophy, 81, 155-174.

Menéndez-Benito, P. (2013). On Dispositional Sentences. In A. Mari, C. Beyssade, \& F. del Prete (Eds.), Genericity (pp. 276-292). Oxford: Oxford University Press.

Molnar, G. (2003). Powers. Oxford: Oxford University Press.

Park, S. (2017). Against extrinsic dispositions. Review of Contemporary Philosophy, 16, 92-103.

Portner, P. (2009). Modality. Oxford: Oxford University Press.

Quine, W. V. (1960). Word and object. Cambridge, MA: MIT Press.

Quine, W. V. (1970). Natural kinds. In D. Davidson \& D. Rescher (Eds.), Essays in Honour of Carl G. Hempel. Dordrecht: D. Reidel.

Silk, A. (2017). Modality, weights and inconsistent premise sets. Journal of Semantics, 34, 683-707.

Steinberg, J. R. (2010). Dispositions and subjunctives. Philosophical Studies, 148, 323-341.

Vetter, B. (2011). On linking dispositions and which conditionals? Mind, 120, 1173-1189.

Vetter, B. (2014). Dispositions without conditionals. Mind, 123, 129-156. 
Vetter, B. (2015). Potentialities: From dispositions to modality. Oxford: Oxford University Press.

Viebahn, E., \& Vetter, B. (2016). How many meanings for 'may'? The case for modal polysemy. Philosopher's Imprint, 16(10), 1-26.

Wellwood, A. (2015). On the semantics of comparison across categories. Linguistics and Philosophy, 38, 67-101.

Wellwood, A., Hacquard, V., \& Pancheva, R. (2012). Measuring and comparing individuals and events. Journal of Semantics, 29, 207-228.

Yalcin, S. (2007). Epistemic modals. Mind, 116, 983-1026.

Yalcin, S. (2010). Probability operators. Philosophy Compass, 5, 916-937.

Yli-Vakkuri, J. (2010). Conditional and habitual analyses of disposition ascriptions. The Philosophical Quarterly, 60, 624-630. 